\title{
Análisis de la integración del enfoque de género en las políticas de cooperación al desarrollo: El caso de las instituciones de la Comunidad Autónoma del País Vasco
}

\author{
IDOYE ZABALA ERRAZTI \\ Instituto Hegoa, UNIVERSIDAD DEL PAÍS VASCO (UPV/EHU), ESPAÑA. E-mail: \\ idoye.zabala@ehu.es
}

MARIA JOSÉ MARTINEZ HERRERO

Instituto Hegoa, UNIVERSIDAD DEL PAÍS VASCO (UPV/EHU), ESPAÑA. E-mail: mjose.martinez@ehu.es

IRATI LABAIEN EGIGUREN
Instituto Hegoa, UNIVERSIDAD DEL PAÍS VASCO (UPV/EHU), ESPAÑA. E-mail:
irati.labaien@ehu.es

RESUMEN

Este artículo se centra en el análisis de la integración del enfoque de género en los documentos de planificación de las políticas de cooperación al desarrollo y más concretamente de la cooperación descentralizada española. Para ello, se plantea un marco de referencia capaz de evaluar el diseño y la coherencia de las políticas, sin plantear por tanto un análisis de los resultados ni del impacto de dichas políticas, teniendo en cuenta las limitaciones en la aplicación de la perspectiva de género de los principales organismos internacionales de cooperación, las características de la cooperación descentralizada y la necesidad de un marco de evaluación de dichas políticas. Posteriormente utilizamos dicho marco para evaluar las políticas de cooperación de las principales instituciones públicas del País Vasco.

Palabras clave: Cooperación descentralizada, perspectiva de género, empoderamiento de las mujeres, transversalización.

\section{Analysis of the Integration of the Gender Perspective in Development Cooperation Policies: The Case of the Basque Institutions}

\begin{abstract}
This article focuses on the analysis of the integration of gender perspective in planning documents of development cooperation policies and more specifically, in Spanish decentralized cooperation policies. For this analysis, we suggest a framework in order to evaluate the design and the coherence of these policies, without taking into account the result and the impact analysis of them, but considering the limitations of the implementation of gender perspective of the main international co-operation organizations, the characteristics of Decentralized Co-operation and the need of an evaluation framework for these policies. Afterwards, we apply this framework for evaluating co-operation policies of the major public institutions of the Basque Country.
\end{abstract}

Keywords: Decentralized Co-operation, Gender Equity, Women Empowerment, Mainstreaming.

Clasificación JEL: B54, F35, I38, O19

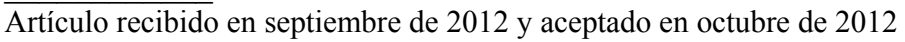

Artículo disponible en versión electrónica en la página www.revista-eea.net, ref. ə-30310 


\section{INTRODUCCIÓN}

La integración del enfoque de género en las políticas de cooperación es un objetivo que adquiere un mayor impulso tras la cuarta conferencia sobre la mujer de Naciones Unidas celebrada en Beijing en 1995. Desde entonces se han dado pasos por parte de las organizaciones y agencias de cooperación para aplicar esta integración utilizando una estrategia dual. Por un lado, incorporar el análisis de género de forma transversal en todas las políticas e intervenciones de la cooperación; por otro, realizar acciones específicas que reduzcan la desigualdad entre los sexos y fomenten el empoderamiento de las mujeres. Más allá del reconocimiento político de la importancia del tema, las organizaciones se han encontrado con dificultades para aplicar de forma efectiva la perspectiva de género en las actuaciones de cooperación.

En este artículo se quiere examinar en qué medida se ha logrado la integración de este enfoque en la cooperación descentralizada en España. Este tipo de cooperación tiene características específicas que la diferencian de la cooperación realizada por el estado español, especialmente su menor dependencia de la política exterior de los estados y su mayor cercanía a la sociedad civil que tiene un mayor peso en la ejecución de las intervenciones de cooperación, por lo que requiere un análisis propio dada la importancia que tiene en nuestro país. Siendo la cooperación de las instituciones de la Comunidad Autónoma del País Vasco ( $C A P V$, a partir de ahora) una de las más relevantes de la cooperación descentralizada, hemos elegido su estudio para aplicar unos criterios de evaluación que nos permitan medir los avances y las limitaciones en la incorporación de la perspectiva de género en las políticas de cooperación.

En las páginas que siguen abordamos distintas cuestiones. En el apartado segundo tratamos de manera resumida los principales debates que se han dado sobre el papel de las mujeres y de las relaciones entre mujeres y hombres en el desarrollo de los países y cómo éstos se han plasmado en distintas estrategias de cooperación.

En el tercer apartado analizamos cómo se produce la integración de la perspectiva de género en la cooperación descentralizada en España a partir del estudio de los documentos doctrinales, tanto de las leyes de cooperación como de los planes directores vigentes de las CCAA para, de esta manera, considerar la necesidad de una pauta para avanzar en la evaluación de dicha integración. Para ello, planteamos a continuación una serie de criterios que permiten dicho análisis evaluativo, pautas que analizan desde la orientación, la pertinencia y la coherencia de la estrategia de género hasta los recursos y los agentes disponibles para ello.

En el cuarto apartado aplicamos dichos criterios para evaluar la incorporación del enfoque de género en la cooperación descentralizada del País Vasco tanto en la Ley de cooperación vasca como en los planes directores y en las 
convocatorias de cooperación de las instituciones objeto de estudio (Gobierno Vasco, Ayuntamientos de Bilbao, Donostia y Vitoria-Gasteiz, y las Diputaciones Forales de Álava, Gipuzkoa y Bizkaia) con el fin de resaltar las principales fortalezas y debilidades de sus políticas.

Finalmente, en el quinto apartado, realizamos unas consideraciones finales a modo de conclusiones sobre el estudio realizado que, partiendo del análisis del caso vasco, se pueden aplicar al conjunto de la cooperación descentralizada.

En anexo damos cuenta de la documentación consultada para este estudio.

\section{IGUALDAD DE GÉNERO Y EMPODERAMIENTO DE LAS MUJERES EN LA COOPERACIÓN AL DESARROLLO}

A lo largo de las últimas décadas, los debates sobre la manera de incorporar el enfoque de género a las estrategias de cooperación se han llevado a cabo de forma paralela a la evolución de los análisis sobre el papel de las mujeres y el de las relaciones de género en los procesos de desarrollo.

Durante todo este tiempo las estrategias de desarrollo se han basado en distintas visiones ${ }^{1}$ del papel que podían jugar las mujeres en los países llamados "en vías de desarrollo". Si bien en un primer momento se puso el énfasis principal en su papel como madres, en lo que se denominó estrategia del bienestar, en los años 70 del siglo pasado el movimiento feminista puso en la agenda la lucha por la igualdad entre mujeres y hombres e impulsó la estrategia de equidad, lo cual tendría su reflejo en las agencias de cooperación con el apoyo de la primera conferencia de NN.UU. sobre la mujer celebrada en México en 1975. La visión liberal de los años 70 impulsó el movimiento Mujeres en el Desarrollo (MED) caracterizado por la búsqueda de la igualdad entre mujeres y hombres, y desde él se plantearon distintas aproximaciones -como la denominada estrategia anti-pobreza- que enfatizaba el rol de las mujeres como productoras para ayudar a salir de su situación de privación. Posteriormente, tras la crisis de la deuda en los años 80 y las políticas de ajuste puestas en marcha, la llamada estrategia de eficiencia fue la que se aplicó a las mujeres de los países pobres, en un contexto de fuertes recortes sociales que afectaron muy negativamente el bienestar de los países donde se aplicaron.

Las estrategias precedentes iban dirigidas en general a diferentes necesidades prácticas de las mujeres, bien como madres, bien como productoras o también como gestoras de las estrategias de supervivencia ante la crisis, pero no tenían una visión global -ni siquiera de sus necesidades prácticas- y no cuestionaban la subordinación que vivían las mujeres. Además, buena parte de ellas tenían un carácter instrumental que buscaba qué podían hacer las mujeres para mejorar la

\footnotetext{
${ }^{1}$ Un análisis histórico de estas visiones se encuentra en Buvinic (1983) y en Moser (1991).
} 
situación de sus países y no tanto qué podían hacer las políticas públicas por ellas. Una excepción fue la estrategia de equidad que sí cuestionó la desigual relación entre hombres y mujeres y la subordinación de estas últimas, aunque sin entrar en otras desigualdades sociales que condicionaban el desarrollo de los países del Sur y las situaciones de mujeres y hombres de esos países (Zabala, 2010). A pesar de estas limitaciones, los enfoques MED contribuyeron a llamar la atención de los organismos y agencias de cooperación sobre la necesidad de incluir a las mujeres en las políticas de desarrollo que se estaban llevando a cabo.

La corriente crítica con el movimiento MED desarrolló el concepto de "relaciones de género" planteando que el problema no eran las mujeres, sino las relaciones sociales construidas social e históricamente entre mujeres y hombres, que delimitan las formas específicas de relación entre ambos géneros y que colocan a las mujeres en posición subordinada. A esta visión crítica se le conoce como enfoque Género en el Desarrollo (GED). Desde los años 80, los movimientos de mujeres de los países del Sur se plantearon un importante cuestionamiento de las políticas de desarrollo existentes y de los distintos enfoques en relación a las mujeres que se habían aplicado previamente y pusieron sobre la mesa una nueva estrategia basada en el empoderamiento de las mujeres ${ }^{2}$.

En este contexto, el análisis de género fue abriéndose paso y adoptándose por las agencias de cooperación y desarrollo, aplicándose como un instrumento de planificación y política. Así, con la celebración de la cuarta Conferencia de Naciones Unidas sobre las Mujer, celebrada en Beijing en 1995, se produjo un compromiso de los gobiernos participantes para conseguir la igualdad de género y el empoderamiento de las mujeres, siendo la transversalidad de género el mecanismo principal para lograr estos objetivos. Desde entonces las principales instituciones internacionales, los gobiernos y la sociedad civil han buscado cómo aplicar este compromiso en sus políticas y actuaciones tanto internas como externas.

La transversalidad de la perspectiva de género es definida según el Consejo Económico y Social de NN.UU. como:

“...el proceso de evaluar las implicaciones para mujeres y hombres de cada acción planificada, incluida la legislación, políticas o programas, en todas las áreas y a todos los niveles. Es una estrategia para hacer de todas las preocupaciones y experiencias tanto de las mujeres como de los hombres una dimensión integral del diseño, la implementación, el seguimiento y la evaluación de las políticas y programas en todas las esferas políticas, económicas y sociales de forma que las mujeres y los hombres se

\footnotetext{
${ }^{2}$ Ver Kabeer (1994) para un análisis crítico de las visiones liberales del enfoque MED y León (1997) para profundizar en el concepto de empoderamiento.
} 
beneficien igualmente y la desigualdad no se perpetúe. El objetivo último es conseguir la igualdad de género" (en Moser y Moser, 2005: 12.). ${ }^{3}$

Este ambicioso objetivo, que supone aplicar la perspectiva de género como una prioridad horizontal que atraviese todas las políticas públicas y todos sus instrumentos, plantea sin embargo algunas dificultades: al ser responsabilidad de todas las instituciones termina no siéndolo de nadie y acaba por quedarse "sin hogar propio"4. Esta consideración estuvo en la base de la necesidad de aplicar una estrategia dual que implica que junto a una prioridad horizontal o transversalidad, se requieren acciones específicas que busquen de forma activa acabar con la desigualdad y fomentar el empoderamiento de las mujeres. Desde esta perspectiva, tanto las principales instituciones internacionales, como la cooperación del gobierno central, o la descentralizada han llevado a cabo diversas propuestas sobre la transversalización y sobre las estrategias duales. Así, el Comité de Ayuda al Desarrollo (CAD) de la OCDE publicó en 1999 las Directrices del CAD sobre Igualdad de Género y Empoderamiento de la Mujer en el marco de la Cooperación para el Desarrollo que sirve de orientación para los donantes. Por su parte, la Unión Europea en su Consenso sobre Desarrollo de la $U E$ de 2005 reconoce como objetivo por derecho propio la igualdad de género, siendo este uno de los cinco principios esenciales de la cooperación al desarrollo, política que ha ido desarrollándose en acuerdos posteriores.

También la cooperación española ${ }^{5}$ elaboró en 2007 una Estrategia de "Género en Desarrollo", estrategia alineada con los mandatos internacionales, y que marca a su vez la adopción de la transversalidad en los planes directores de las Comunidades Autónomas (CC.AA.) y de buena parte de sus leyes de cooperación. En el caso de la Comunidad Autónoma del País Vasco la consideración de la equidad de género como prioridad transversal se generalizó en las instituciones desde comienzos de la década de 2000, y en especial a partir de la Ley Vasca de Igualdad de 2005 en donde se recomienda la transversalidad en todas las políticas y programas.

Las principales referencias existentes para establecer las políticas de género de las instituciones son la Convención para la Eliminación de Todas las Formas de Discriminación contra la Mujer (CEDAW) aprobada en 1979, y la Plata-

\footnotetext{
${ }^{3}$ Traducción propia.

${ }^{4}$ Tal como señala el PNUD (2004).

${ }^{5}$ Con el mandato de la Ley orgánica (3/2007) para la igualdad efectiva de mujeres y hombres que aborda el tratamiento como prioridad transversal y específica en el ámbito de la cooperación y el mandato de elaborar una estrategia sectorial de igualdad para la cooperación española. La Estrategia elaborada pretende dar cumplimiento a esta Ley de Igualdad y a las prioridades de género que establece el Plan Director de Cooperación Internacional para el Desarrollo (Ley 23/1998).
} 
forma de Acción de Beijing (1995) ${ }^{6}$. Tras la Declaración del Milenio (2000), la consecución de los Objetivos de Desarrollo del Milenio (ODM), especialmente el ODM 3 sobre empoderamiento de las mujeres o el ODM 5 sobre salud materna, ha sido adoptada como referencia explícita por instituciones y gobiernos. Por su parte, los principios expuestos en la Declaración de París de 2005 sobre la Eficacia de la Ayuda rigen como marco para mejorar la calidad de la ayuda oficial.

Ahora bien, si el principal avance se ha producido en la adopción de políticas de igualdad de género en la cooperación al desarrollo, lo que ha conllevado la existencia de unidades de género en muchas de las instancias encargadas de cooperación, lo cierto es que estas políticas han tendido a diluirse a la hora de su puesta en práctica. Como se ha señalado, el sesgo masculino implícito en los valores, ideología, intereses materiales, organización y procedimientos impregna a las agencias de desarrollo lo que provoca la evaporación de las políticas de género (Longwe, 1997). De entre los factores ${ }^{7}$ que favorecen la evaporación, caben destacar: la falta de capacitación del personal en temas de género; la cultura de la organización, incluyendo resistencias a la idea de igualdad de género; el tratamiento del tema de forma aislada lo que no ayuda a que sea transversal al conjunto de actuaciones; y la falta de sentimiento de apropiación de la política (Moser y Moser, 2005).

La existencia de estos límites es reconocida en distintos documentos de las principales organizaciones internacionales de cooperación ${ }^{8}$, aunque se buscan medidas concretas que ayuden a superarlos. La adopción de los ODM como agenda de desarrollo y la aplicación de la Declaración de París no han ayudado a mejorar la perspectiva de género de la cooperación ${ }^{9}$, aunque en los últimos años se ha buscado como evitar sus principales carencias. En este sentido, la

${ }^{6}$ Otras referencias que utilizan las agencias y organizaciones de cooperación son el Programa para la Acción de El Cairo (1994) o la Resolución 1325 sobre Mujeres, Paz y Seguridad (2000).

${ }^{7}$ Extraídos de una evaluación realizada a 14 instituciones y organizaciones entre las que había donantes bilaterales, instituciones financieras internacionales, agencias de NN.UU. y ONGD.

${ }^{8}$ El CAD confirma que sus miembros tienen políticas de igualdad de género, pero que muy pocos tienen personal, presupuestos y prácticas de gestión que permitan poner en marcha la política y rendir cuentas de la misma (OCDE/CAD, 2007). La Unión Europea señala que las principales barreras para implementar su enfoque de política están en la falta de conciencia de los temas de género en los niveles de decisión, la escasez de recursos humanos y presupuestarios para esas tareas y la falta de pericia en género (European Commission, 2010).

${ }^{9}$ Una crítica más detallada de los problemas derivados de la adopción de los ODM y de la Eficacia de la Ayuda se puede encontrar en Zabala y Martínez (2012). También Karen Oppenheim señala que según aquellas agencias que llevan más tiempo trabajando el tema, los cambios en las modalidades de ayuda tras la Declaración de París presentan más dificultades que oportunidades para lograr la igualdad de género (OCDE/CAD, 2007). Nava San Miguel (2009) realiza un análisis de los retos y oportunidades de la agenda de género y eficacia de la ayuda a nivel internacional. 
Agenda de Acción de Accra, acordada en el Tercer Foro sobre la Eficacia de la Ayuda y la declaración de Partenariado Global por la Eficacia de la Cooperación para el Desarrollo firmada en el Cuarto Foro de Busan suponen un avance en el reconocimiento de la importancia de la igualdad de género.

Por su parte, la cooperación española no es ajena a estos límites, que se reflejan en las dificultades para integrar el enfoque de género a pesar de los avances en materia de adopción del tema desde el II Plan Director 2005-2008 con un carácter de doble prioridad o enfoque dual a través de la transversalidad (prioridad horizontal) y como sector específico, y del importante aumento del presupuesto dedicado a género ${ }^{10}$. Algunos análisis sitúan el problema en la escasa institucionalización del tema en las estructuras de cooperación, lo que estaría dificultando la implementación del enfoque GED en las actuaciones llevadas a cabo, las cuales tenderían a reproducir más bien el enfoque MED (Perez, 2008).

A modo de resumen, destacaremos la existencia de algunos límites y carencias que han sido señalados en lo que afecta a la adopción de la transversalización del enfoque de género. Entre ellas estarían las siguientes:

- Falta de suficientes capacidades en materia de género, tanto en las instituciones, como en las ONGD y en las contrapartes.

- Ausencia de políticas institucionales de igualdad de género en los organismos y agencias de cooperación, incluyendo incentivos, respaldo de alto nivel para este objetivo y recursos humanos y económicos.

- Dificultades en el diálogo político con los países socios sobre igualdad de género con participación de la sociedad civil, incluidas las organizaciones de mujeres.

- Insuficiente y deficiente ${ }^{11}$ utilización del marcador de género del $\mathrm{CAD}^{12}$ para conocer la ayuda dedicada específicamente a la igualdad de género y el empoderamiento de las mujeres que también es adoptado por la $\mathrm{UE}^{13}$.

\footnotetext{
${ }^{10}$ Para un análisis de los avances y límites en la integración del enfoque de género en la cooperación española ver Tomás (2010).

${ }^{11}$ No se discrimina entre las acciones con un enfoque MED dirigidas a mejorar la situación de las mujeres y las que pretenden cambiar las relaciones entre hombres y mujeres y mejorar su posición, que tienen un enfoque GED.

${ }^{12}$ Este marcador indica si en un proyecto la equidad de género es objetivo fundamental, marcador principal; si es un objetivo dentro de otros, marcador significativo; o si no es objetivo del mismo, marcador no orientado al objetivo (OCDE/CAD, 2012). Actualmente los 24 miembros del CAD aplican el marcador que cubre el $75 \%$ de la ayuda asignada a sectores $(90 \%$ si excluimos a EE.UU.) (O’Neill, 2012).

${ }^{13}$ European Commission, 2010, donde se plantea que para 2015 se aplique al menos al $80 \%$ de todos los proyectos de la Unión Europea.
} 
- Escasa incorporación de los indicadores de igualdad de género y empoderamiento de las mujeres en los marcos de resultados de las agencias, para lo que se necesita también recopilar y analizar datos desagregados por $\operatorname{sexo}^{14}$.

- Ausencia de procedimientos, normas y regulaciones que permitan una gestión orientada a resultados en esta materia. Es importante, por ejemplo, conseguir compromisos plurianuales en los donantes ${ }^{15}$.

Estos y otros límites y carencias en la integración de la perspectiva de género en la política de las agencias y organismos de cooperación han dado lugar en los últimos años a nuevos análisis y propuestas tanto por parte del CAD como de la $\mathrm{UE}^{16}$. En lo que respecta a nuestro trabajo, el mismo se ha centrado precisamente en el estudio de cómo y hasta qué punto la cooperación descentralizada ha adoptado un enfoque de género de acuerdo con las líneas internacionales en la materia y qué problemas se han suscitado al respecto para, a partir de ahí, discutir los criterios que pueden servir para evaluar la incorporación de la perspectiva de género en esta política.

\section{LA INTEGRACIÓN DEL ENFOQUE DE GÉNERO EN LA COOPERACIÓN DESCENTRALIZADA DEL ESTADO ESPAÑOL}

Es importante señalar que la cooperación descentralizada es un rasgo singular de la Ayuda Oficial al Desarrollo (AOD) española representando un 14,7\% de promedio de la AOD total entre 2002 y 2008 (Martínez y Sanahuja, 2010), siendo el país que más ayuda descentralizada realiza de entre los países del CAD. Además, partiendo de los criterios del $\mathrm{CRS}^{17}$ la importancia relativa de la AOD descentralizada destinada a género es aún mayor, llegando a alcanzar en 2006 el 35\% del total de la AOD española destinada a género, aunque este porcentaje disminuyera en los años siguientes, tal y como apunta Alioska Perez

${ }^{14}$ Tal como recomienda la Agenda de Acción de Accra (23a) y recoge el CAD (OCDE/CAD, 2008).

${ }^{15}$ Lograr objetivos de igualdad de género y empoderamiento supone la existencia de procesos que requieren tiempo, por lo que los proyectos plurianuales son más adecuados.

${ }^{16}$ Mecanismos reflejados en los últimos documentos como el Plan de Acción sobre Igualdad de Género y Empoderamiento de las Mujeres en el Desarrollo 2010-2015 (European Commission, 2010), los Principios Rectores del CAD en materia de Eficacia de la Ayuda, Igualdad de Género y Empoderamiento de la Mujer (OCDE/CAD, 2008) y la Gestión orientada a resultados en materia de igualdad de género en los organismos donantes de la Red sobre Igualdad de Género del CAD (GENDERNET) (OCDE/CAD, 2009).

${ }^{17}$ Credit Reporting Sistem es la referencia del CAD para agrupar la ayuda por sectores. Posteriormente el CAD ha desarrollado un conjunto de marcadores entre los que está el marcador de género que permite un mejor seguimiento de la transversalización de la perspectiva de género. 
$(2008)^{18}$, como consecuencia del importante crecimiento de la ayuda dedicada a este tema por parte de la cooperación central. Por otra parte, uno de los rasgos que caracteriza a la cooperación descentralizada es la mayor proximidad a los sectores más activos de la sociedad y su papel en el aumento de la conciencia ciudadana, lo que afecta también a los movimientos de mujeres y su relación con las políticas de cooperación. Todo ello hace especialmente interesante el análisis de la cooperación descentralizada desde esta perspectiva, cuestión que se desarrolla en este apartado.

\subsection{La integración de la perspectiva de género en los documentos doctrinales de las Comunidades Autónomas}

En el caso concreto de las Comunidades Autónomas nos interesamos por la importancia dada a la equidad de género en el momento de la concepción y diseño de sus políticas de cooperación y para ello tenemos en cuenta las leyes de cooperación así como los últimos planes directores vigentes en cada una de las CC.AA., además de las convocatorias de subvenciones publicadas durante la vigencia de los respectivos planes. Desde estas bases, analizamos en un primer momento, el tratamiento y el rango que alcanza la equidad de género en los documentos analizados -si es objetivo o elemento inspirador, si es objetivo transversal o incluso prioridad transversal- para posteriormente estudiar los mecanismos dispuestos para lograr los objetivos previstos.

Por lo que se refiere a las leyes de cooperación autonómica, Canarias, Cantabria, Castilla y León, País Vasco, Illes Balears, la Comunidad Valenciana y el Principado de Asturias incluyen la equidad de género como una prioridad transversa $^{19}$, mientras que todos los planes directores ${ }^{20}$, insertan la transversalidad de género de manera uniforme. En cuanto a sectores de actuación, llama la atención que la inclusión de la equidad de género y/o el empoderamiento de las mujeres como prioridad sectorial se da en mayor medida en las Leyes de cooperación $^{21}$ (14 CCAA) que en los propios Planes Directores (10 CCAA)

${ }^{18}$ Perez, Alioska (2008) “Los avances para la integración del enfoque de género en la política de desarrollo de la cooperación descentralizada en España, 2005-2007. Fundación Carolina, Madrid; es una referencia clave, a nivel del estado español, a la hora de analizar la cooperación descentralizada desde una perspectiva de género.

${ }^{19}$ La ausencia de esta transversalidad en otras CC.AA. quizás se deba a que la ley de igualdad e incluso el Plan Estratégico de Género son posteriores a las leyes de cooperación. Sin embargo, en todas las leyes aparece como principio aunque no se especifique la transversalidad.

${ }^{20}$ Salvo Murcia que no tiene Plan Director.

${ }^{21}$ En el caso de Madrid y Extremadura no son prioridades sectoriales sino áreas de actuación preferentes. 
puesto que no es considerada como tal en los de Aragón, Canarias, Cantabria ${ }^{22}$, Galicia, Extremadura y La Rioja.

El tratamiento dado al enfoque de género, tanto en las leyes de cooperación como en los planes directores, no es homogéneo en todas las CC.AA. En ocasiones se realiza una aproximación desde el enfoque de derechos o desde la igualdad de oportunidades de las mujeres, en otras desde el empoderamiento de las mujeres y su participación equitativa en los procesos de desarrollo. Asimismo, observamos que aunque algunas leyes de cooperación presentan deficiencias en cuanto al tratamiento dado al género como transversal, la equidad de género o el empoderamiento de las mujeres como sector de actividad tiene mayor presencia que en los planes directores. Es el caso de Aragón, Extremadura y La Rioja que no incluyen este sector en sus planes directores mientras que está bien explicitado en sus leyes de cooperación.

\section{Figura 1}

La equidad de género o el fortalecimiento de las capacidades de las mujeres como prioridad sectorial en los Planes Directores de las CC.AA.

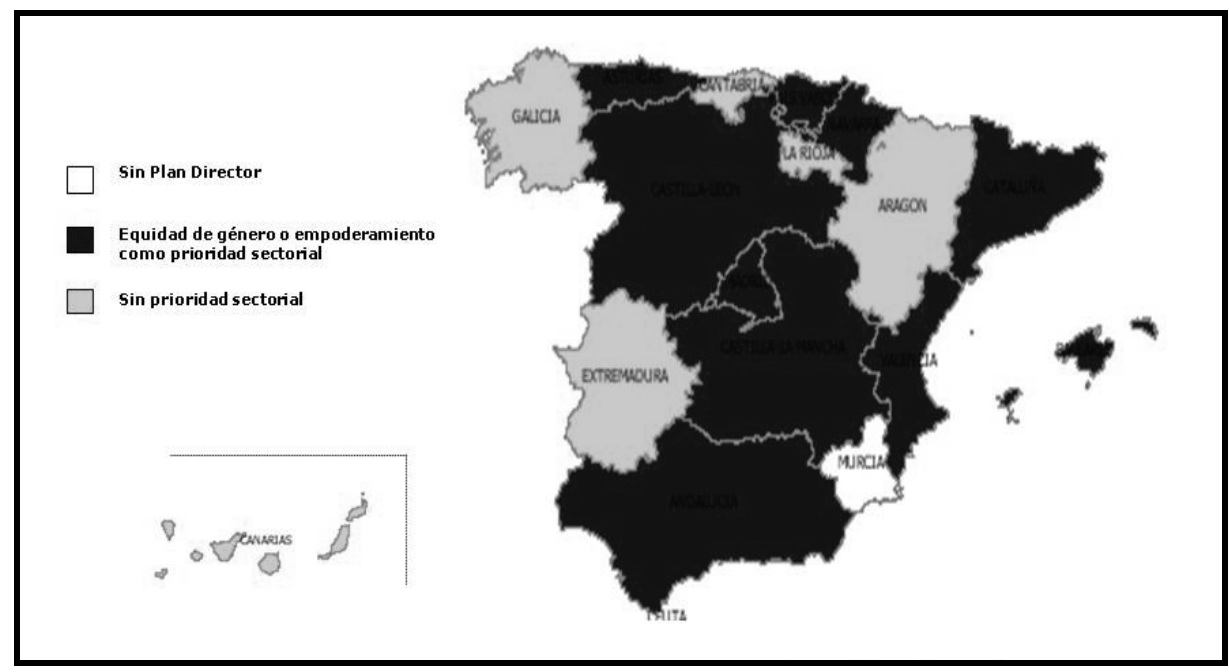

Fuente: Elaboración propia.

En definitiva, podemos decir que, de manera general, las CCAA tienen en cuenta las referencias y recomendaciones tanto internacionales como nacionales en cuanto a la perspectiva de género ya que además de ser una transversal (en los planes directores mayoritariamente), la equidad de género y el empoderamiento de la mujer se considera una prioridad sectorial (en los planes y en las

${ }^{22}$ Cantabria no lo define expresamente pero lo introduce dentro de otras prioridades sectoriales. 
leyes de cooperación). Estas referencias refuerzan la intencionalidad de los gobiernos autonómicos de considerar el género como un elemento primordial a la hora de planificar las políticas de cooperación.

Una vez analizada la manera en que se contempla la transversalidad y la sectorialidad en el plano doctrinal, nos hemos interesado por conocer los instrumentos aplicados para poner en práctica el discurso teórico. Para ello, tendremos en cuenta las convocatorias, decretos y órdenes que las Comunidades Autónomas han publicado durante la vigencia del Plan Director analizado y, más concretamente, los criterios de valoración de las convocatorias ${ }^{23}$.

En ocasiones nos encontramos que la equidad de género como prioridad transversal no aparece en los baremos (Andalucía, Aragón, Canarias, y Castilla y León) aunque, a pesar de no evaluar la transversalidad en los criterios, se menciona de una manera informativa previamente en las convocatorias realizadas durante la vigencia de los planes directores ${ }^{24}$. En estas CCAA la ausencia de la equidad de género en los baremos es un problema ya que si no se valora como prioridad transversal su aplicación se diluye. El resto de Comunidades incluyen la transversalidad como característica a valorar en al menos una de las convocatorias publicadas. La transversalidad no siempre se expande a todas las líneas de subvención ni a todos los agentes de cooperación; en general, en las intervenciones de cooperación (proyectos o programas) se valora la transversalidad aunque no siempre se incluye en ayuda humanitaria o en educación para el desarrollo (es el caso de Asturias, Castilla-La Mancha, Extremadura, Islas Baleares, Madrid, La Rioja, Navarra y Valencia). En ocasiones, los baremos establecidos varían en función de los agentes encargados de la cooperación e incluso desaparecen cuando es la propia administración quien realiza las intervenciones conveniadas.

En cuanto a la baremación del empoderamiento de las mujeres como prioridad sectorial, nueve CC.AA. la integran de forma parcial al menos en una de las convocatorias publicadas o en una línea específica (Castilla y León, Castilla-La Mancha, Cataluña, Extremadura, Galicia, La Rioja, Madrid, Navarra y País $\left.\operatorname{Vasco}^{25}\right)$.

En conclusión, el discurso sobre la equidad de género y/o el empoderamiento de las mujeres está presente en los documentos doctrinales de las

\footnotetext{
${ }^{23}$ No todas las convocatorias especifican los criterios de valoración, incluso a veces son diferentes para cada modalidad.

${ }^{24}$ Este hecho también se da en las convocatorias de Cantabria y Cataluña.

${ }^{25}$ En la CAPV no se barema la sectorialidad, pero en la baremación de la transversalidad se incluyen las acciones de empoderamiento de las mujeres.
} 
CC.AA.; algunas agencias y direcciones han realizado estrategias específicas ${ }^{26}$, e incluso, se han adoptado compromisos presupuestarios destinados a acciones de género (País Vasco, Cataluña y Navarra) aunque no siempre se especifica la naturaleza de este porcentaje, es decir, si se dedica a fomentar la transversalización o la sectorialización. Además se han dado avances a nivel doctrinal aunque aun hay que hacer un esfuerzo en los criterios de valoración. Tal y como señala Alioska Pérez (2008), en los aspectos más formales de la política de cooperación se han ido adoptando con distinto énfasis las políticas de igualdad de género en un sentido transversal ${ }^{27} \mathrm{y}$, en menor medida, en las políticas sectoriales orientadas al empoderamiento y al aumento de capacidades de las mujeres.

En todo caso, el análisis realizado muestra la inexistencia de consensos o ideas compartidas sobre la manera de aplicar -más allá de las propuestas doctrinales- las estrategias de género en la cooperación descentralizada, sin que existan tampoco pautas concretas que sirvan para evaluar esta política. En nuestra opinión, ello representa un hándicap importante a la hora de analizar y discutir la manera de avanzar en este terreno. Por ello, en el siguiente apartado se plantean algunos criterios que pueden servir de referencia para realizar dicho análisis evaluativo.

\subsection{Criterios para evaluar la incorporación de la perspectiva de género en las políticas de cooperación descentralizada}

Para avanzar hacia un marco de referencia que sirva para profundizar en el análisis de la integración del enfoque de género en las políticas de cooperación descentralizadas hemos partido de tres tipos de consideraciones. En primer lugar, las que se derivan de las limitaciones ya apuntadas en el apartado 2 y que tienen que ver con las propuestas y recomendaciones de diversas agencias y organismos tanto de la cooperación multilateral como bilateral. En segundo término, los aspectos relativos a las características propias de la cooperación descentralizada para lo que hemos tenido en cuanta la propuesta metodológica contenida en Unceta et al. ${ }^{28}$, adaptándola a nuestro fin. Y por último, las propias observaciones realizadas sobre la orientación concreta de las estrategias de co-

\footnotetext{
${ }^{26}$ Estrategia Asturiana de Género para la Cooperación al Desarrollo (2010), Directrices de equidad entre los hombres y las mujeres de la Agencia Catalana de Cooperación al Desarrollo (2011).

${ }^{27}$ Cabe destacar el avance y la influencia que ha tenido en este sector la Estrategia de "Género en Desarrollo" elaborado por la DGPOLDE en el año 2007. Los debates impulsados desde la Red GEDEA (Género en Desarrollo y Eficacia de la Ayuda) también han contribuido a los avances en este sentido.

${ }^{28}$ El marco de referencia queda reflejado en el libro de Unceta et al. (2011): La Cooperación al Desarrollo Descentralizada: Una propuesta metodológica para su análisis y evaluación. Hegoa, UPV/EHU.
} 
operación en materia de género de las CC.AA., a las que nos hemos referido en el subapartado anterior (3.1.).

Partiendo de todo ello consideramos que es posible identificar algunos criterios específicos de análisis, los cuales hemos agrupado en torno a cuatro grandes bloques:

\section{a) Definición y orientación de la estrategia dual:}

- Análisis del grado de definición y alcance de la transversalización del enfoque de género en toda la política y las actuaciones planteadas, lo cual afecta tanto a los instrumentos (subvenciones para proyectos y programas, cooperación directa y convenios), como a las modalidades de cooperación (proyectos y programas de cooperación al desarrollo sobre el terreno; sensibilización y educación para el desarrollo; y ayuda humanitaria y de emergencia)

- Análisis de las prioridades y objetivos específicos relativos a la superación de las desigualdades de género y a favorecer el empoderamiento de las mujeres en las distintas intervenciones desplegadas y en los sectores de intervención contemplados. Ello supone considerar no sólo la existencia del empoderamiento de las mujeres como posible prioridad sectorial específica, sino también su inclusión y análisis en otras líneas sectoriales contempladas.

\section{b) Coherencia de la estrategia:}

- Análisis de los instrumentos e incentivos dispuestos para la aplicación de la estrategia diseñada, lo que se traduce especialmente en la consideración de baremos que valoren la adecuación de las intervenciones. Es una cuestión bastante general que los baremos se apliquen a las actuaciones que realizan los agentes de cooperación a través de su inclusión en las convocatorias para proyectos o programas y en menor medida en las modalidades de ayuda humanitaria o educación para el desarrollo, pero resulta menos común que las propias instituciones se apliquen a sí mismas o a los convenios que aprueban fuera de las convocatorias los mismos baremos.

- Análisis de la existencia de partidas presupuestarias especificas que impulsen la equidad de género y el empoderamiento de las mujeres lo que resulta asimismo fundamental para que el discurso no se diluya por falta de medios y pueda hacerse efectivo el compromiso adquirido en dicha materia. Estos recursos financieros han de ser previsibles de forma que los agentes implicados puedan conocerlos con antelación y ajustar sus acciones en materia de equidad de género. 


\section{c) Recursos para la gestión de la estrategia:}

- Análisis de los equipos humanos de gestión contemplados y de su cualificación en materia de cooperación al desarrollo, incluyendo los aspectos relativos a la continuidad de su trabajo, así como la existencia de personas o unidades especializadas en temas de género que garanticen la inclusión de la perspectiva de género en todas las intervenciones. Se considera asimismo que las áreas de igualdad de las distintas instituciones de las CC.AA. pueden jugar un papel importante en la transversalización del género si trabajan de forma coordinada con las personas responsables de cooperación.

- Análisis de los recursos disponibles por parte de los equipos de gestión, los cuales se consideran imprescindibles tanto para las tareas de diseño, como para las de seguimiento y evaluación del trabajo realizado. En este ámbito resultan fundamentales sistemas de información bien diseñados, compatibles y homologados que puedan aportar información estadística desagregada por género, así como indicadores que permitan dar seguimiento y evaluar los avances realizados.

\section{d) Agentes encargados de llevar a cabo la estrategia:}

- Análisis de los distintos tipos de agentes contemplados para impulsar y llevar a cabo los objetivos planteados. Ello incluye los diagnósticos sobre las capacidades institucionales y sociales existentes, y las previsiones sobre la participación en la estrategia de organizaciones de mujeres y grupos feministas, universidades, ONGD, o de las propias administraciones y sus unidades de género o de igualdad.

- Análisis de las capacidades y limitaciones de las ONGD en esta materia y mecanismos previstos para superarlas. Este asunto adquiere gran relevancia ya que hasta al momento, la mayor parte de las instituciones autonómicas y locales han basado su estrategia en las ONGD, lo que ha puesto de manifiesto algunas dificultades para la incorporación del enfoque de género.

\section{ANÁLISIS DE LA INCORPORACIÓN DE LA PERSPECTIVA DE GÉNERO EN LA COOPERACIÓN DESPLEGADA POR LAS INSTITUCIONES DE LA CAPV}

En este último apartado, y a la luz de los criterios reseñados más arriba, se examina la incorporación de la perspectiva de género en el caso de las instituciones de la CAPV, lo que como ya se ha dicho tiene un especial interés dada la importante aportación que, tanto en el plano doctrinal como en la práctica, se ha llevado a cabo desde esta comunidad. En este análisis de caso hemos tenido en 
cuenta la documentación más relevante desde los inicios de la cooperación vasca hasta la actualidad.

Para ello, nos hemos centrado específicamente en una serie de instituciones (el Gobierno Vasco, las Diputaciones de Álava, Bizkaia y Gipuzkoa, y los Ayuntamientos de Bilbao, Donostia y Vitoria-Gasteiz), por ser las que cuentan con un mayor bagaje tanto en el ámbito del diseño de políticas -todas ellas poseen planes directores de cooperación- como en el de la aplicación de las mismas. El importante papel que han jugado en el campo de la cooperación a lo largo de 25 años queda reflejado tanto en las aportaciones financieras como en la variedad de las intervenciones realizadas (más de 850 millones de euros para financiar cerca de 9.000 actividades distintas). Los resultados obtenidos son fruto del estudio de los diferentes documentos doctrinales y de política elaborados a lo largo de estos años por dichas instituciones. A continuación se presenta un breve resumen de dichos resultados, de los que se han extraído los aspectos más relevantes.

\subsection{Definición y orientación de la estrategia dual: Transversalidad y sectorialidad}

Podemos señalar en primer lugar que todas las instituciones estudiadas han realizado un esfuerzo por incorporar los temas transversales como objetivos o prioridades, tal y como se recomienda desde los organismos y agencias internacionales. Además, la equidad de género es la línea transversal que con mayor profundidad se ha tratado y la que ha logrado un alto grado de concreción en las políticas de cooperación vasca. Tanto el Gobierno Vasco como las Diputaciones y los Ayuntamientos analizados, incluyen desde los años noventa los temas de género en todos sus documentos así como en los baremos y en las convocatorias realizadas. Si bien en un primer momento la preocupación por estas cuestiones se reflejó más tímidamente, con el tiempo fue tomando mayor protagonismo, al calor del impulso y desarrollo legislativo que se ha dado a la igualdad entre mujeres y hombres en todos los ámbitos de la política y de la sociedad. En la Tabla 1 puede verse la secuencia temporal en que dicho tema fue mencionado, considerado como eje transversal, y objeto de baremación.

Ya en 1993 el Ayuntamiento de Vitoria-Gasteiz hacía una alusión temprana a la situación de las mujeres en los países empobrecidos y el Gobierno Vasco, a través del Fondo de Cooperación al Desarrollo (FOCAD) ${ }^{29}$, reflejaba en su convocatoria de 1996 la preocupación por incorporar la perspectiva de género tanto en la formulación como en la ejecución y evaluación de sus intervenciones, llegando en 2002 a incluir una clausula de exclusión directa para todo pro-

${ }^{29}$ El FOCAD recoge las aportaciones del Gobierno Vasco y las Diputaciones Forales de Álava, Bizkaia y Gipuzkoa para intervenciones de cooperación al desarrollo. 
yecto que no contemple la perspectiva de género, al considerarse que ninguna acción a favor del desarrollo es neutra en esta materia.

Con posterioridad, tanto la ley vasca de Cooperación al Desarrollo de 2007 como los planes directores elaborados por las distintas instituciones han tratado de manera extensa la transversalidad de género desde una perspectiva de desarrollo humano en sentido amplio, siendo la igualdad de mujeres y hombres uno de los principios orientadores de la política y debiendo estar presente en todas las acciones de forma transversal además de como prioridad sectorial ${ }^{30}$. El Gobierno Vasco contempla la transversalidad de género en sus planes directores, incluyendo medidas positivas y manteniendo la exclusión de los proyectos que no incorporen un análisis de género. Además, el segundo Plan Director (20082011) dedica un objetivo sectorial al empoderamiento de las mujeres resaltando la participación de los hombres en la construcción de un nuevo modelo social más justo y equitativo. Asimismo la equidad de género es transversal en la ayuda humanitaria y en la educación para el desarrollo, mostrando un apoyo e impulso claro a las organizaciones de mujeres y al movimiento feminista.

Tabla 1

La equidad de género en los documentos doctrinales de la cooperación vasca

\begin{tabular}{|l|c|c|c|}
\hline \multirow{2}{*}{} & \multicolumn{3}{|c|}{ PRIMERA CONSIDERACIÓN COMO } \\
\cline { 2 - 4 } & MENCIÓN & TRANSVERSAL & BAREMO \\
\hline Gobierno Vasco & 1996 & $2000\left(^{*}\right)$ & 2000 \\
\hline Diputación de Álava & 1999 & 2000 & 1999 \\
\hline Diputación de Bizkaia & 2001 & 2001 & 2001 \\
\hline Diputación de Gipuzkoa & 2004 & $2004\left(^{* *}\right)$ & 2004 \\
\hline Ayuntamiento. Vitoria-Gasteiz & 1993 & & 1993 \\
\hline Ayuntamiento de Bilbao & $2005\left(^{* * *}\right)$ & 2006 & 2005 \\
\hline Ayuntamiento de Donostia & 2008 & 2008 & 2008 \\
\hline
\end{tabular}

(*) No aparece como transversal, pero desde 1999 los decretos del Gobierno Vasco incluyen la "relación con objetivos transversales" al referirse a la baremación de las transversales.

$\left.{ }^{* *}\right)$ En la DFG se plantea en los baremos desde 2004, considerándose prioridad de actuación aunque no como línea transversal.

$\left.{ }^{* * *}\right)$ En 2001, su convocatoria se refería a priorizar actuaciones que contribuyan a "mejorar la situación de la mujer, de las poblaciones indígenas y de los niños", pero sin mencionar la perspectiva de género.

Fuente: Elaboración propia.

Por lo que se refiere a las Diputaciones, en el Plan Director (2009-2011) elaborado de forma conjunta por las mismas, la equidad de género no sólo es línea transversal en los proyectos de cooperación sino también en la sensibilización y educación para el desarrollo. Cabe destacar la perspectiva feminista que se per-

\footnotetext{
${ }^{30}$ Hay que tener en cuenta que la Ley Vasca de Igualdad entre mujeres y hombres data de 2005.
} 
cibe en este documento puesto que se realiza un extenso y profundo análisis de las desigualdades de género planteando la necesidad de establecer unas relaciones más justas y equitativas entre mujeres y hombres mediante el empoderamiento de las mujeres y la deconstrucción masculina patriarcal en el caso de los hombres, implicándoles así de forma activa en la lucha por la equidad de género. Asimismo se debe resaltar las referencias a los derechos de los colectivos de lesbianas, gays, transexuales y bisexuales. Estas aportaciones representan el rasgo diferenciador de este plan director respecto a los del resto de las instituciones.

En cuanto a los Ayuntamientos, en los dos planes directores del Ayuntamiento de Bilbao (2006-2009) y (2010-2013) se introduce la equidad de género como línea transversal y el empoderamiento de las mujeres como área sectorial incluyendo, en el resto de áreas sectoriales, actuaciones específicas orientadas a potenciar el papel y la participación de las mujeres y a satisfacer sus necesidades. El Ayuntamiento de Donostia, en su Plan Director (2008-2011), plantea que la equidad de género es una de las cinco líneas transversales, mientras que en la transversal de derechos humanos se especifican los de las mujeres y los hombres, y en la sostenibilidad medioambiental la equidad de género se plantea como una prioridad. A nivel sectorial, aunque ninguna de las 14 áreas se dedica a cuestiones de género, 6 sectores aluden a la equidad, a las mujeres o a la perspectiva de género. Por último, el Ayuntamiento de Vitoria-Gasteiz, pionero en este tema ${ }^{31}$, en su Plan Director de Vitoria-Gasteiz (2010-2013) da un giro a su actuación en materia de cooperación al considerar que no es realista plantear la transversalidad de género como requisito absoluto, es decir excluyente, siendo más operativo priorizar las actuaciones orientadas a acciones específicas de empoderamiento de mujeres y de igualdad entre mujeres y hombres.

En la Tabla 2 se muestra de manera conjunta la consideración transversal y sectorial de la problemática de género en los documentos de política de las instituciones estudiadas.

Tabla 2

La equidad de género y los planes directores de las instituciones de la CAPV

\begin{tabular}{|l|c|l|l|}
\hline & \multicolumn{3}{|c|}{ PLANES DIRECTORES } \\
\cline { 2 - 4 } & $\begin{array}{c}\text { Transversal/ } \\
\text { Sectorial }\end{array}$ & \multicolumn{1}{|c|}{$\begin{array}{c}\text { Transversal de otras líneas } \\
\text { transversales }\end{array}$} & \multicolumn{1}{c|}{ Prioridad sectorial } \\
\hline Gobierno Vasco & $2005 / 2008$ & $\begin{array}{l}\text { Ayuda Humanitaria } \\
\text { Educación al desarrollo }\end{array}$ & Empoderamiento de mujeres \\
\hline Diputación de Álava & $2009 / 2009$ & $\begin{array}{l}\text { Sensibilización y educación al } \\
\text { desarrollo }\end{array}$ & $\begin{array}{l}\text { Empoderamiento de las mujeres y } \\
\text { deconstrucción masculina patriarcal }\end{array}$ \\
\hline Diputación de Bizkaia & $2009 / 2009$ & $\begin{array}{l}\text { Sensibilización y educación al } \\
\text { desarrollo }\end{array}$ & $\begin{array}{l}\text { Empoderamiento de las mujeres y } \\
\text { deconstrucción masculina patriarcal }\end{array}$ \\
\hline
\end{tabular}

${ }^{31}$ En su I Plan Municipal de Acción Positiva para las Mujeres de 1999 incluía de forma expresa la integración de la perspectiva de género en las políticas y programas de cooperación 
Tabla 2 (continuación)

La equidad de género y los planes directores de las instituciones de la CAPV

\begin{tabular}{|l|c|l|l|}
\hline & \multicolumn{3}{|c|}{ PLANES DIRECTORES } \\
\cline { 2 - 4 } & $\begin{array}{c}\text { Transversal/ } \\
\text { Sectorial }\end{array}$ & \multicolumn{1}{|c|}{$\begin{array}{c}\text { Transversal de otras líneas } \\
\text { transversales }\end{array}$} & \multicolumn{1}{c|}{ Prioridad sectorial } \\
\hline Diputación de Gipuzkoa & $2009 / 2009$ & $\begin{array}{l}\text { Sensibilización y educación al } \\
\text { desarrollo }\end{array}$ & $\begin{array}{l}\text { Empoderamiento de las mujeres y } \\
\text { deconstrucción masculina patriarcal }\end{array}$ \\
\hline Ayto. de Vitoria-Gasteiz & $----/ 2010$ & & $\begin{array}{l}\text { Empoderamiento e igualdad entre } \\
\text { mujeres y hombres }\end{array}$ \\
\hline Ayto. de Bilbao & $2006 / 2006$ & $\begin{array}{l}\text { Actuaciones específicas en todas } \\
\text { las áreas }\end{array}$ & Empoderamiento de mujeres \\
\hline Ayto. de Donostia & $2008 / 2008$ & $\begin{array}{l}\text { Derechos humanos } \\
\text { Sostenibilidad medioambiental }\end{array}$ & Cuestiones de género \\
\hline
\end{tabular}

Fuente: Elaboración propia.

\subsection{Coherencia de la estrategia: Baremos y previsiones presupuestarias}

En todas las instituciones analizadas existe una preocupación por la coherencia de la política con perspectiva de género en la cooperación, tal y como se constata en los baremos y en los criterios de exclusión presentes en las convocatorias de proyectos de cooperación, de sensibilización y educación para el desarrollo y, en menor medida, en ayuda humanitaria y de emergencia.

El Gobierno Vasco desde el año 2000 plasma la transversalización de la equidad de género en los criterios de baremación de las intervenciones de cooperación. Con pequeñas variaciones y según los años, se establecen baremos para acciones en proyectos de cooperación y productivos o en educación para el desarrollo y acción humanitaria, y además se valoran positivamente aquellas iniciativas tanto de organizaciones vascas y/o de contrapartes que tengan un plan estratégico pro-equidad de género.

El Plan Director de las diputaciones forales refleja en su baremación los derechos de las mujeres; así se valora la información desagregada por sexos, el tratamiento de la posición de las mujeres y el incremento de su poder en relación a sus derechos económicos, sociales, culturales, civiles, políticos, o sexuales y reproductivos ${ }^{32}$, además de la coordinación entre los movimientos feministas del norte y del sur, o la creación y/o consolidación de redes. Las cuestiones de género se incluyen también en la baremación de las otras dos directrices (desarrollo humano local y educación para el desarrollo). Finalmente cabe destacar el maltrato a las mujeres o la defensa de personas perseguidas por su orientación sexual en los criterios específicos de cada diputación.

\footnotetext{
${ }^{32}$ Se distinguen entre los proyectos dirigidos especialmente a mujeres y los que tratan de garantizar sus derechos considerando otros aspectos como el entorno institucional o el trabajo con la población masculina. Además la no desagregación de la población por sexo se penaliza con una reducción de puntos en la baremación del proyecto.
} 
Los ayuntamientos también incentivan la transversalización en sus baremos. Así el Ayuntamiento de Bilbao, a través de las convocatorias de intervención, evalúa la perspectiva de género en la calidad técnica y coherencia del proyecto, la política de género de las ONGD solicitantes y su capacidad para trabajar en este campo, la experiencia de las contrapartes en el tema o en la población protagonista de la intervención. El Ayuntamiento de Donostia, en su plan director, propone unos criterios de baremación coherentes con la importancia atribuida al enfoque de género como línea transversal. Por último, el Ayuntamiento de Vitoria-Gasteiz desde 2003 adopta una estrategia más pragmática, estableciendo dos tipos de proyectos: los generales sin excesivas exigencias de género y los especiales que suponen un mayor compromiso con la equidad de género y el empoderamiento de las mujeres y a los que se premia en la baremación y en la asignación económica (Murguialday 2008). A pesar de ello, en las convocatorias exige un análisis del impacto de género con medidas correctoras y advierte que la obtención de cero puntos en algunos apartados supone la eliminación del proyecto.

Sólo dos de las instituciones analizadas incluyen una previsión presupuestaria específica que garantice la perspectiva de género en sus actuaciones. El Gobierno Vasco, en el primer Plan Director de 2005, establecía que al menos un $10 \%$ del presupuesto disponible se destinara a acciones dirigidas exclusivamente a mujeres, organizaciones de mujeres o a proyectos, con el objetivo explícito del empoderamiento de las mujeres y/o la mejora en los niveles de equidad de género ${ }^{33}$; mientras que en la evaluación del II Plan Director se señala que el porcentaje aumentó hasta un 14,7\% (Agencia Vasca de Cooperación, 2011). Por su parte el Ayuntamiento de Bilbao establece unos porcentajes mínimos que garanticen la perspectiva de género en los fondos destinados a la cooperación y a la educación para el desarrollo.

\subsection{Recursos para la gestión de la estrategia: Equipos humanos y sistemas de información}

Más allá de los fondos dedicados, las instituciones responsables de llevar adelante las políticas de cooperación han contado con medios escasos, especialmente teniendo en cuenta lo ambicioso de los objetivos planteados, lo que contrasta con el mayor presupuesto de gestión que tienen otras políticas (Unceta et al., 2012). Además, los continuos cambios de localización dentro del organigrama institucional, motivados muchas veces por los cambios políticos, han dificultado la continuidad en la gestión de la cooperación.

33 Además se indica que el $50 \%$ de la población beneficiaria directa de las acciones impulsadas por el Gobierno Vasco sean mujeres y busca que se fortalezca el movimiento de mujeres. 
En el caso del Gobierno Vasco, este contraste entre la dotación de fondos para cooperación y la cantidad dedicada a la gestión de los mismos se concreta en la temporalidad y precariedad en la contratación de personal técnico. Los primeros años la gestión recae en personas sin experiencia en el campo de la cooperación siendo más tarde cuando se incorporen personas cualificadas, aunque en calidad de becarias muchas de ellas. Con la creación de la unidad de género en el año 2008, se incorpora una técnica más que compatibiliza su trabajo como responsable de género con el resto de las tareas de la Dirección de Cooperación, quedando así reducida su dedicación al tema.

La puesta en marcha de la Agencia Vasca de Cooperación supone un avance en cuanto a personal ${ }^{34}$. La creación de una unidad de género dentro de la Agencia mejorará el seguimiento de las iniciativas de la Dirección de Cooperación orientadas a fomentar la igualdad entre mujeres y hombres aplicando la perspectiva de género en los proyectos y programas. Sin embargo, está por ver si su trabajo va a contar con suficientes apoyos para realizar el trabajo de transversalización de género tanto dentro de la Agencia como en su trabajo de cooperación.

Las diputaciones, para su gestión, optaron por equipos externos cualificados de carácter externo que, desde 2007, comparten tareas con el personal técnico en cooperación de cada institución. Por el contrario, los ayuntamientos cuentan con personal propio que con los años ha ido cualificándose y aumentando en número, dando mayor estabilidad a la gestión. Además en Bilbao y Donostia cooperación e igualdad comparten la misma Área generándose sinergias entre las personas que gestionan la igualdad de género y la cooperación.

Emakunde, como instituto vasco de la mujer, participa en el Consejo Asesor de Cooperación al Desarrollo de Euskadi desde 1999 con el objetivo de aportar una perspectiva de género a los programas de cooperación al desarrollo $\mathrm{y}$, a partir de 2008, en el nuevo Consejo Vasco de Cooperación en donde, además, se promueve la representación equilibrada de mujeres y hombres en atención a la legislación vasca vigente sobre igualdad (2005) y sobre cooperación (2007).

Los sistemas de información constituyen un punto débil de la cooperación vasca puesto que las instituciones analizadas utilizan categorías distintas para los sectores de intervención. El problema más grave en cuanto a perspectiva de género es que, por una parte, cada proyecto solo puede asignarse a un sector, lo que dificulta la valoración de la transversalidad de género en los proyectos y, por otra parte, la mayor parte de la información de las instituciones carece de un sector de empoderamiento de las mujeres, "Los sistemas de información utilizan un único descriptor para cada intervención, lo que impide valorar proyectos multisectoriales en los que el objetivo de género tenga una presencia significa-

\footnotetext{
${ }^{34}$ La Agencia cuenta con seis técnicos/as en cooperación y uno/a en igualdad de género.
} 
tiva" (Unceta et al., 2012). Además, en numerosas ocasiones se deja al libre albedrío de las entidades beneficiarias la definición del sector de actuación; e incluso finalmente, cada institución cuando define el sector, utiliza una clasificación diferente. Por ese motivo, las diputaciones han optado por un sistema que permite el seguimiento del número y monto de las intervenciones que tienen como objetivo central la defensa de los derechos de las mujeres, al margen del sector al que pertenezca cada intervención.

Por último, la inexistencia de unos sistemas de información suficientes y homogeneizados dificultan las posibilidades de evaluación de la política de cooperación en general y los aspectos de género en particular ${ }^{35}$. Esto enlaza con la necesidad de establecer indicadores de género que puedan medir los cambios realizados tanto dentro de las instituciones y de los agentes como en las actividades, principalmente proyectos y programas que se realizan.

\subsection{Agentes encargados de llevar a cabo la estrategia}

En este punto cabe destacar la escasa diversificación existente en la cooperación vasca puesto que las ONGD ocupan un lugar principal, mientras que otros agentes sociales como colectivos profesionales, empresas, sindicatos, universidades u organizaciones de mujeres participan en ella de forma esporádica.

La importancia alcanzada por la transversalidad de género se debe, en gran parte, a la participación en el mundo de la cooperación de mujeres con trayectoria feminista, del grado de sensibilidad y de conocimiento del tema de algunas personas responsables de las instituciones que se ha reflejado en los documentos programáticos de la política de cooperación, y del importante trabajo del Grupo de Género de la Coordinadora de ONGD de Euskadi. De hecho, la cooperación pública vasca constituye hoy en día una referencia en el conjunto de las CC.AA. en la incorporación de la perspectiva de género. (Pérez, 2008; Viadero y Alberdi, 2011).

Sin embargo, muchos agentes de cooperación han acabado por asociar las transversales con referencias específicas sobre algunos temas, con el objetivo de que sus proyectos superen los criterios de baremación establecidos por las instituciones. En el caso concreto de la perspectiva de género, se dan situaciones poco coherentes dada la dificultad y la escasa capacidad que poseen las ONGD tanto para la formulación de propuestas como para su ejecución sobre el terreno.

\footnotetext{
${ }^{35}$ En la práctica resulta difícil dar seguimiento a cualquier aspecto relacionado con los sectores, dada la variedad de situaciones en el tratamiento sectorial de las instituciones. Hemos observado la utilización de distintas categorías para los sectores de intervención; así el Gobierno Vasco y el Ayuntamiento de Bilbao utilizan las categorías del CAD, el Ayuntamiento de Vitoria-Gasteiz las de AECID, y el Ayuntamiento de Donostia y las diputaciones tienen categorías propias.
} 
De entre las limitaciones que encuentran las ONGD para incorporar la perspectiva de género a sus organizaciones y a sus propuestas destacamos, en primer lugar, el desconocimiento del propio significado puesto que, para muchas ONGD, género es lo mismo que mujeres (Volio, 2004); algunas piensan que género supone considerar los problemas de las mujeres o las diferencias entre hombres y mujeres, pero muy pocas consideran la perspectiva de género como un instrumento capaz de superar las desigualdades de poder entre ambos sexos (Murguiaday et al., 2000). Además, y en segundo lugar, buena parte de las ONGD consideran la perspectiva de género más como una imposición de las instituciones que algo asumido como propio; “...la mayoría dicen haber llegado a esto por imposición de la Dirección de Cooperación y no porque estuviesen previamente convencidas de su importancia" (Volio, 2004:10). A pesar del esfuerzo por superar los aspectos formales, el resultado es que las propuestas carecen de suficiente coherencia en materia de género en buena parte de los proyectos.

Para superar estas limitaciones y fortalecer la visión de género, el Grupo de Género de la Coordinadora de ONGD organiza talleres formativos, reuniones, evaluaciones, etc. con el objetivo de fortalecer el enfoque de género a nivel organizativo. Una medida que cabe resaltar por ser la primera comunidad autónoma que la lleva a cabo es que desde 2008 las propias instituciones ponen en marcha un instrumento de fortalecimiento institucional, con partida presupuestaria específica en cada año, orientado a promover los procesos de cambio organizacional pro-equidad de género en aquellas organizaciones que lo deseen, tanto vascas como del sur. A pesar de ello, se requiere tiempo y dinero de las propias ONGD para que estas propuestas sean suficientes para fortalecer una política de género.

Para avanzar hacia una aplicación de las políticas de cooperación con perspectiva de género es importante incorporar entidades sociales de mujeres y organizaciones feministas, tanto de la CAPV como del Sur, dada la experiencia y cualificación que poseen en el trabajo por la equidad de género y el empoderamiento de las mujeres. A pesar de las propuestas realizadas desde distintos ámbitos, por el momento los resultados son más bien exiguos ya que son pocas las organizaciones de mujeres que han participado en ellas.

Por último, hay que señalar el papel de las propias instituciones vascas en la cooperación descentralizada y su capacidad para implicar a recursos técnicos y personal de otros departamentos, áreas o servicios de la propia administración. En este sentido, los departamentos de cooperación que comparten Área con el de igualdad o el de la mujer, han fomentado y generado una serie de sinergias que facilitan esta implicación. 


\section{CONSIDERACIONES FINALES}

Podemos señalar que la cooperación descentralizada en general y la vasca en particular comparten fortalezas y debilidades con las registradas por las instituciones internacionales. Entre las fortalezas destaca el avance producido en la importancia que se otorga a la equidad de género y al empoderamiento de las mujeres a través de la transversalidad de la perspectiva de género en la cooperación descentralizada y la puesta en marcha de acciones específicas, aunque como hemos visto este avance es desigual en las CC.AA. La falta de medidas concretas para aplicar esta perspectiva es la principal debilidad, lo que implica que se sigue produciendo una evaporación de la política de género lo que se constata en que buena parte de las CC.AA. no tienen unidades de género, ni presupuestos específicos, ni indicadores que permitan el seguimiento y la evaluación de las políticas.

En el caso de la CAPV se han ido tomando algunas medidas de acuerdo con las que se quieren aplicar a nivel internacional, aunque todavía no han dado los resultados esperados. El establecimiento de baremos en el conjunto de las instituciones y de previsiones presupuestarias en algunas de ellas es un paso positivo para que las políticas diseñadas no se diluyan. Podemos destacar como positiva la búsqueda del aumento de las capacidades de los agentes implicados a través de una mayor formación en género, asesorías, e incluso la promoción de políticas institucionales pro-equidad de género que afectan a las ONGD y, previsiblemente, a la Agencia Vasca de Cooperación.

Entre las recomendaciones que creemos son necesarias para superar las debilidades existentes en la cooperación vasca vemos necesario fortalecer los equipos de gestión en materia de cooperación y, específicamente, en materia de género así como unos mejores sistemas de información armonizados, lo que permitiría un mayor seguimiento y evaluación de las intervenciones a través de indicadores de equidad de género y empoderamiento de las mujeres que ayuden a discernir si estas actuaciones tiene un enfoque dirigido a resolver algunas necesidades prácticas de las mujeres, mejorando su situación (lo que conocemos como enfoque "mujer en el desarrollo" o enfoque MED), o si tienen una vocación de transformación en las relaciones entre mujeres y hombres buscando acabar con la subordinación femenina (o enfoque "género en el desarrollo" GED). Esto exige la creación o consolidación de unidades de género con personas especialistas en la materia que cuenten con recursos en términos económicos y de tiempo.

Deben realizarse esfuerzos en la dirección de implicar a otros agentes sociales con mayor capacidad y pericia en la lucha por transformar las relaciones de género como son los grupos de mujeres y feministas tanto vascos como de los países con los que se coopera para que se establezcan redes de colaboración que beneficien a sus participantes. 
También ha de darse una mayor cooperación y coordinación entre las propias instituciones vascas de cooperación. Todas ellas, en mayor o menor medida, han hecho esfuerzos en incorporar la perspectiva de género en su trabajo y tienen experiencias y buenas prácticas que podrían compartir, lo que les permitiría una mayor implicación en la cooperación directa. Esta mayor coordinación permitiría, al mismo tiempo, una mejor especialización que supondría mejorar la eficiencia de la cooperación descentralizada.

Consideramos, finalmente, que es necesaria la aplicación de un marco de referencia para evaluar la incorporación de la perspectiva de género en la cooperación descentralizada. Sin ánimo de ser exhaustivos, los criterios planteados en este trabajo y aplicados a la evaluación de la cooperación de las instituciones de la CAPV pueden ayudar a realizar este análisis.

\section{REFERENCIAS BIBLIOGRÁFICAS}

AGENCIA VASCA DE COOPERACIÓN (2011): Evaluación participativa del Plan Estratégico y Director para el Desarrollo 2008-2011. Kalidadea.

BUVINIC, M. (1983): "Women's issues in Third World Poverty: A policy analisis" en M. Buvinic, Margaret A. Lycette y William Paul McGreevey, Women and Poverty in the Third World, pp. 14-33. The Johns Hopkins University Press, Baltimore.

EUROPEAN COMMISSION (2010): EU Plan of Action on Gender Equality and Women's Empowerment in Development: 2010-2015. Commission Staff Working Document, SEC(2010) 265 final.

KABEER, N. (1994): Reversed realities: Gender hierarchies in development thought. Verso, London.

LEÓN, M. (comp.) (1997): Poder y empoderamiento de las mujeres. Edit. Tercer Mundo, Bogotá.

LONGWE, S. H. (1997): "The evaporation of gender policies in the patriarchal cooking pot" en Development in Practice, Vol.7, n 2, pp. 148-156.

MAEC (2007): Estrategia de "Género en Desarrollo" de la cooperación española, Secretaría de Estado de Cooperación Internacional, Dirección General de Planificación y Evaluación de Políticas para el Desarrollo.

MARTÍNEZ, I. y SANAHUJA, J. A. (2010): "La cooperación descentralizada en España y el reto de la eficacia de la ayuda" en Icei Paper, $\mathrm{n}^{\circ} 18$. Instituto Complutense de Estudios Internacionales.

MOSER, C. (1991): "Las mujeres en la planificación del desarrollo. Necesidades prácticas y estratégicas de género" en Políticas de cooperación para el desarrollo y participación de las mujeres, pp. 11-40. Hegoa, Bilbao. 
MOSER, C. y MOSER, A. (2005): "Gender mainstreaming since Beijing: A review of success and limitations in international institutions" en Gender and Development, Vol. 13, $\mathrm{n}^{\circ}$ 2, pp. 11-22.

MURGUIALDAY, C.; DEL RÍO, A.; ANITUA, E. y MAOÑO, C. (2000): Perspectiva de género en las ONGD vascas. Mugarik Gabe, Hegoa.

MURGUIALDAY, C. (2008): La equidad de género en la cooperación al desarrollo del Ayuntamiento de Vitoria-Gasteiz: balance de una década y propuestas. Mimeo

OCDE/CAD (2007): Igualdad de género y la entrega de la ayuda oficial al desarrollo: ¿Qué ha cambiado en las agencias de cooperación para el desarrollo desde 1999?

OCDE/CAD (2008): Principios rectores del CAD en materia de eficacia de la ayuda, igualdad de género y empoderamiento de la mujer.

OCDE/CAD (2009): Resumen temático 4: Gestión orientada a resultados en materia de igualdad de género en los organismos donantes. Red sobre igualdad de género del CAD (Gendernet).

OCDE/CAD (2012): Aid in Support of Gender Equality and Women's Empowerment. February 2012. Disponible en: http://www.oecd.org/dac/ aidstatistics/49732892.pdf [8-8-12].

O'NEILL, P. (2012): "Follow the money - Tracking financing for gender equality" en UN Commission on the status of women, 1 march 2012. Financing for gender equality - Panel 2. Disponible en: http://www.oecd.org/dac/ aidstatistics/50273454.pdf [8-8-12].

PEREZ, A. (2008): Los avances para la integración del enfoque de género en la política de desarrollo de la cooperación descentralizada en España, 20052007. Fundación Carolina, Madrid.

PNUD (2004): Transforming the Mainstream: Gender in UNDP. Bureau for Development Policy. Nueva York.

SAN MIGUEL, N. (2009): "Oportunidades y propuestas en la agenda de género y eficacia. Avanzando hacia 2015" en Estefanía Molina y Nava San Miguel: Nuevas líneas de investigación en Género y Desarrollo. Cuadernos Solidarios, $n^{\circ}$ 3. UAM Ediciones, Madrid.

TOMÁS, R. (2010): "Una mirada al enfoque de género de la cooperación española: Avances y cambios frente a viejos y nuevos desafíos internacionales" en 200 años de Iberoamérica. Congreso Internacional 1810-2010, pp. 27572776.

UNCETA, K. (dir.); GUTIÉRREZ, J.; LABAIEN, I.; MALAGÓN, E.; MARTÍNEZ, $\mathrm{M}^{a}$ J.; SABALZA, M.; VILLENA, U. y ZABALA, I. (2011): La cooperación al desarrollo descentralizada: una propuesta metodológica para su análisis y evaluación. Hegoa- UPV/EHU. Bilbao.

UNCETA, K. (dir.), AMIANO, I.; GUTIÉRREZ, J.; LABAIEN, I.; MALAGÓN, E.; MARTÍNEZ, M ${ }^{\text {a }}$ J.; SABALZA, M.; VILLENA, U. y ZABALA, IDOYE (2012): 25 años de Cooperación al Desarrollo en Euskadi: La política de las institu- 
ciones públicas y el sistema vasco de cooperación. Hegoa- UPV/EHU. Bilbao.

VIADERO, M. y ALBERDI, J. (2011): "La incorporación de la participación y la equidad de género en las cooperaciones autonómicas" en Cuadernos de Hegoa, $n^{\circ} 54$, Hegoa. Bilbao.

VOLIO, R. (2004): Diagnóstico de género de la convocatoria de 2000 del FOCAD. Mimeo.

ZABALA, I. (2010): "Estrategias alternativas en los debates sobre Género y Desarrollo" en Revista de Economía Crítica n 9, pp. 75-89.

ZABALA, I. y MARTÍNEZ, Ma J. (2012): “Una visión de género de la agenda de desarrollo y de la eficacia de la ayuda", en Internacionalización en tiempos de crisis, XIV Reunión de Economía Mundial, Jaén, 30 de mayo al 1 de junio. 


\section{Anexo: Documentación consultada}

\section{A) Leyes de Cooperación}

Asamblea de Extremadura (2003): LEY 1/2003, de 27 de febrero, de Cooperación para el Desarrollo.

Asamblea de Madrid (1999): LEY 13/1999, de 29 de abril, de Cooperación para el Desarrollo de la Comunidad de Madrid.

Asamblea Regional de Murcia (2007): LEY 12/2007, de 27 de diciembre, de Cooperación Internacional para el Desarrollo de la Comunidad Autónoma de la Región de Murcia.

Congreso de los Diputados (1998): LEY 23/1998, de 7 de julio, de Cooperación Internacional para el Desarrollo.

Cortes de Aragón (2000): LEY 10/2000, de 27 de diciembre, relativa a la Cooperación para el Desarrollo.

Cortes de Castilla La Mancha (2003): LEY 3/2003, de 13 de febrero de 2003, de Cooperación Internacional para el Desarrollo.

Cortes de Castilla y León (2006): LEY 9/2006, de 10 de octubre, de Cooperación al Desarrollo.

Cortes Valencianas (2007): LEY 6/2007, de 9 de febrero, de la Cooperación al Desarrollo de la Comunitat Valenciana.

Junta General del Principado de Asturias (2006): LEY 4/2006, de 5 de mayo, de Cooperación al Desarrollo.

Parlament de Catalunya (2001): LEY 26/2001, de 31 de diciembre, de Cooperación al Desarrollo.

Parlament de les Illes Balears (2005): LEY 9/2005, de 21 de junio, de Cooperación para el Desarrollo.

Parlamento de Andalucía (2003): LEY 14/2003, de 22 de diciembre, de Cooperación Internacional para el Desarrollo.

Parlamento de Canarias (2009): LEY 4/2009, de 24 de abril, Canaria de Cooperación Internacional para el Desarrollo.

Parlamento de Cantabria (2007): LEY de Cantabria 4/2007, de 4 de abril, de Cooperación Internacional al Desarrollo de la Comunidad Autónoma de Cantabria.

Parlamento de Galicia (2003): LEY 3/2003, de 19 de junio, de Cooperación para el Desarrollo.

Parlamento de La Rioja (2002): LEY 4/2002, de 1 de julio, de Cooperación para el Desarrollo.

Parlamento de Navarra (2001): LEY Foral 5/2001, de 9 de marzo, de Cooperación al Desarrollo.

Parlamento Vasco (2007): LEY 1/2007, de 22 de febrero, de Cooperación para el Desarrollo. 


\section{B) Planes Directores de Cooperación}

Agencia Andaluza de Cooperación Internacional al Desarrollo (AACID) (2008): Plan Andaluz de Cooperación para el Desarrollo 2008-2011, Junta de Andalucía.

Agència Catalana de Cooperació al Desenvolupament (2011): Pla Director de Cooperació al Desenvolupament 2011-2014, Departament de la Presidència, Generalitat de Catalunya.

Agència de Cooperació Internacional de les Illes Balears (2008): Pla Director de la Cooperació de les Illes Balears 2008-2011, Direcció General de Cooperació, Conselleria de d'Afers Socials, Promoció i Immigració, Govern de les Illes Balears.

Asamblea de Extremadura (2010): Plan General de la Cooperación Extremeña 2010-2013, Junta de Extremadura.

Consejo Asturiano de Cooperación al Desarrollo (2009): Plan Director de Cooperación al Desarrollo 2009-2012, Consejería de Bienestar Social y Vivienda, Gobierno del Principado de Asturias.

Departamento de Vivienda y Asuntos Sociales (2008): Plan Estratégico y Director Cooperación para el Desarrollo 2008-2011, Gobierno Vasco.

Dirección General de Acción Social y Cooperación Internacional (2009): Plan Estratégico de Cooperación para el Desarrollo Horizonte 2011, Consejería de Salud y Bienestar Social, Gobierno de Castilla-La Mancha.

Dirección General de Asuntos Europeos y Cooperación al Desarrollo (2009): Plan Director de Cooperación para el Desarrollo de la Comunidad Autónoma de Cantabria 2009-2012, Consejería de Empleo y Bienestar Social, Gobierno de Cantabria.

Dirección General de Inmigración y Cooperación al Desarrollo (2008): Plan Director de la Cooperación Valenciana 2008-2011, Conselleria de Solidaridad y Ciudadanía, Generalitat Valenciana.

Dirección General de Inmigración, Cooperación para el Desarrollo y Voluntariado (2009): Plan General de Cooperación al Desarrollo 2009-2012 de la Comunidad de Madrid, Comunidad de Madrid.

Dirección General de Participación Ciudadana, Acción Exterior y Cooperación (2012): Plan Director de la Cooperación Aragonesa para el Desarrollo (borrador) 2012-2015, Departamento de Presidencia y Justicia, Gobierno de Aragón.

Dirección General de Políticas Migratorias y Cooperación al Desarrollo (2009): Plan Director de Castilla y León de Cooperación al Desarrollo 2009-2012, Consejería de Interior y Justicia, Junta de Castilla y León.

MAEC-AECID: I Plan Director de la Cooperación Española 2001-2004; II Plan Director de la Cooperación Española 2005-2008; III Plan Director de la Cooperación Española 2009-2012. 
Servicio de Acción Exterior y Cooperación al Desarrollo (2008): /l Plan Director de Cooperación de La Rioja 2008-2011, Dirección General de Acción Exterior, Gobierno de La Rioja.

Servicio de Cooperación Internacional (2011): Il Plan Director de la Cooperación Navarra 2011-2014, Consejería de Asuntos Sociales, Familia, Juventud y Deporte, Navarra.

Subdirección General de Cooperación Exterior (2010): II Plan Director de la Cooperación Gallega para el Desarrollo 2010-2013, Dirección General de Relaciones Exteriores y con la UE, Xunta de Galicia.

Vicepresidencia de Emigración y Cooperación con América Latina y Dirección General de Relaciones con África (2010): Plan Director de la Cooperación Canaria para el Desarrollo 2010-2013, Comisionada de Acción Exterior, Gobierno de Canarias.

\section{C) Documentación Consultada Comunidad Autónoma del País Vasco}

\section{a) Planes directores:}

Ayuntamiento de Vitoria-Gasteiz: Plan Director de Cooperación al Desarrollo 2010-2013.

Ayuntamiento de Donostia: Plan Municipal de Cooperación y Educación para el Desarrollo 2008-2011.

Ayuntamiento de Bilbao: I Plan Director de Cooperación al Desarrollo 20062009; II Plan Director de Cooperación al desarrollo 2010-2013.

Diputaciones Forales de Álava, Bizkaia y Gipuzkoa: Plan Director de Cooperación al Desarrollo 2009-2011.

Gobierno Vasco: Plan Director de Cooperación al Desarrollo 2005-2008. Plan Estratégico y Director de Cooperación al Desarrollo 2008-2011.

b) Decretos, convocatorias, bases reguladoras de subvenciones de las siete instituciones vascas analizadas (Gobierno Vasco; Diputaciones Forales de Álava, Bizkaia y Gipuzkoa; Ayuntamientos de Bilbao, Donostia y Vitoria-Gasteiz). 
